\title{
Predicting Centrifugal Compressor Off-design Operation in an Operator Training Simulator
}

\author{
J. Fredrik Nyholm \\ Neste Engineering Solutions, Finland, fredrik.nyholm@neste.com
}

\begin{abstract}
Operator training simulators (OTS) are a valuable tool for improving the competence of both process operators and engineers. As pumps, compressors and turbines are vital parts of any process plant, accurate modeling of these machines is crucial for a dynamic process simulator. The behavior of the machines is usually described by operating curves provided by the manufacturer. However, these curves are strictly valid only for the specific process conditions they were created for. Accurate prediction of the operation outside of these specific conditions may present a formidable challenge. To further improve the accuracy the NAPCON Simulator, a new dynamic modeling method was developed for the centrifugal compressors. The stability of the developed model was verified by dynamic ramp tests of the operating conditions. Furthermore, the accuracy of the developed model was determined by performing process data tests. The results were promising and, in most cases, an improvement from the performance of the currently implemented model.
\end{abstract}

Keywords: dynamic simulation, operator training simulator, centrifugal compressors, off-design operation, exit flow coefficient

\section{Introduction}

Main features of a training simulator include sophisticated models of all process units with fast simulation of various operating states and fault scenarios. A well-designed OTS allows extensive and customizable training functionality. High-fidelity process models that predict accurate thermal performance and chemical conversion for complex process equipment ensure realistic process response. The process model is complemented by automation models containing process controls, safety functions and sequence loops. This all is presented to the trainee by a comprehensively emulated DCS interface complete with trends and alarms, providing a realistic process control experience. (Qvist et al, 2018)

NAPCON Simulator is based on an in-house software called ProsDS, which is programmed in ANSI Common Lisp. The simulator features a library of all commonly used process equipment. The high-fidelity process feedback is achieved by basing the simulation on physical phenomena and chemical reactions. The process equipment is connected by advanced piping network modeling with two-phase flow calculations. The process is modeled on hierarchical flowsheets. Equipment states as well as connectivity can be examined even during active simulation. Calculation steps in the process and automation models of less than one second provide accurate results of the dynamic simulation. The information both inside as well as between the process units is updated at each simulation interval.

In ProsDS the pumps are modeled using theoretical maximum head at zero flow and pressure loss due to flow. The head $H_{\max }$ can be given either as pressure difference or height of fluid column. The pressure loss is modeled using the equation for the valve flow coefficient $C V\left(\mathrm{~m}^{3 *} \mathrm{~s}^{-1} * \mathrm{~Pa}^{-0.5}\right)$, which relates the pressure loss $\Delta p(\mathrm{~Pa})$ to the volumetric flow $\dot{V}\left(\mathrm{~m}^{3} / \mathrm{s}\right)$ as a quadratic function. The $C V$ value is calculated with Equation 1, where $S G(-)$ is the specific gravity of the pumped fluid. The operating curve for the pump is thus given by Equation 2. A curve of this sort is usually accurate enough for pumps around their normal operating points. Changes in the rotating speed affect the head of the pump quadratically. (Nyholm, 2020)

$$
\begin{gathered}
C V=\dot{V} \sqrt{\frac{S G}{\Delta p}} \\
H=H_{\max }-S G \frac{\dot{V}^{2}}{C V^{2}}
\end{gathered}
$$

The compressors are currently modeled as pumps with some extra features. For compressor curve fitting a correction function is used, which takes the actual load of the compressor into account. A correction factor for the effect of guide vanes is also available. Functions for these correction factors are modeled individually for each compressor by the person doing the modeling work. Any other calculations regarding the operating curves are usually carried out as external calculations modeled outside of the machine. No automatic curve correction for changes in e.g. molecular weight or inlet temperature exists in the simulator, these are defined by the modeler as external calculations. For compressors in general, the curve type should be steeper at higher loads 
than what the $C V$-based quadratic curve is. (Nyholm, 2020)

The power requirement of the machines is also modeled in a simple manner. It is based on the flow and pressure difference over the machine, with a corresponding efficiency. The outlet temperature is also modeled in a simple way, based on either an empirically approximated constant or on the inlet and outlet pressures. (Nyholm, 2020)

\section{Compressor theory}

The centrifugal compressor consists of an impeller inside a casing, an inlet leading the gas to the impeller and an exit leading out from the volute surrounding the impeller. The impeller may also be surrounded by a vaned diffuser, but the diffusers in the machines used in the oil refining and petrochemical industries usually come without vanes. If the machine consists of multiple compression stages, the gas is led from the exit, through deswirling vanes, into the inlet of the next impeller. The energy of the gas is increased by increasing its speed, which is done by the impeller. The diffuser and volute then convert the speed to a pressure and temperature increase by efficiently reducing the fluid velocity.

The compressor is characterized by its performance map, where the produced head (or discharge pressure), efficiency and required power are plotted against the inlet volume flow. The manufacturer usually provides a number of performance maps valid for varying inlet conditions. The compressor has an operating range defined between the surge point and choke point.

Off-design operation of centrifugal pumps based on a performance curve can typically be described with reasonable accuracy by the affinity laws. (Pettersson $e t$ $a l, 1990)$. Even if centrifugal compressors and pumps share many similarities both in operating principles and construction, the compressors are more complex machines and the affinity laws are not sufficient to accurately describe a large operating regime for them.

In dynamic simulation the machine is subjected to a wide range of operating conditions and, thus, the operating curve of the machine should be scaled smoothly in a user-friendly manner to match the governing conditions, while maintaining a sufficient accuracy of the predicted performance. As directly scaling the performance curves with the affinity laws is not viable for compressors, approaches that consider the vital machine and operating condition parameters influencing the compressor performance are needed for an accurate prediction of new performance curves. One option is the computationally heavy CFD simulation of the machine. Another approach would be to utilize empirical correlations for the predictions. However, both methods require detailed information of the machine geometry, which usually is hard to come by.

Converting the performance curve to a dimensionless form, from which performance curves at new operating conditions can be predicted, appears to be the most viable option for an accurate implementation in an OTS. Using this method, the head is converted into the head coefficient $\psi(-)$. For the volume flow either the inlet flow coefficient $\varphi_{1}(-)$ or the exit flow coefficient $\varphi_{3}(-)$ is used. Using the inlet flow coefficient, an array of dimensionless curves is obtained and, as the impeller tip speed Mach number varies, the needed dimensionless curve can be interpolated or extrapolated from the curve array and the converted back to the sought performance curve in natural scale. The aim of utilizing the exit flow coefficient is to obtain a single dimensionless performance curve for the compressor, which can then be used to calculate all the curves in natural scale. The advantage with both methods is that only the manufacturer performance curves, impeller diameter and blade tip height and knowledge of the governing process conditions are needed for the predictions. As both the impeller diameter and blade tip height are typically provided in the datasheet of the machine, the difficulties of obtaining information of the detailed geometry of the machine can be avoided.

The presented inlet flow coefficient method requires the impeller diameter and the performance maps of the manufacturer, as well as the reference conditions for these, for transforming the curves to the dimensionless variant. The governing process conditions are needed for estimation of the new performance curves. The impeller tip speed Mach number $M_{u}(-)$ can be calculated by Equation 3, where $U(\mathrm{~m} / \mathrm{s})$ is the blade tip speed, given by Equation 4 . The impeller diameter $d(\mathrm{~m})$ and angular velocity $\omega(1 / \mathrm{s})$ are used for calculating $U$. Additionally, the isentropic exponent $\kappa(-)$, compressibility $Z(-)$, temperature $T(\mathrm{~K})$, universal gas constant $R\left(\mathrm{~J}^{*} \mathrm{kmol}^{-1} * \mathrm{~K}^{-1}\right)$ and gas molecular weight $M W(\mathrm{~kg} / \mathrm{kmol})$ are needed for calculating the Mach number. The index 1 refers to the compressor inlet. If two sets of inlet conditions have equivalent Mach numbers, their dimensionless performance curves will also be the same.

$$
\begin{gathered}
M_{u}=\frac{U}{\sqrt{\kappa_{1} Z_{1} T_{1} \frac{R}{M W}}} \\
U=\pi d \omega
\end{gathered}
$$

Conversion of the performance curves themselves is done by transforming the polytropic head into the dimensionless head coefficient $\psi$ and the volumetric flow into the inlet flow coefficient $\varphi_{1}$, as described by Equations 5 and 6 , where $H_{p}(\mathrm{~J} / \mathrm{kg})$ is the polytropic head.

$$
\begin{aligned}
\psi & =\frac{2 H_{p}}{U^{2}} \\
\varphi_{1} & =\frac{4 \dot{V}}{\pi d^{2} U}
\end{aligned}
$$

After the conversion, an array of dimensionless operating curves, each corresponding to a certain Mach 
number, has been obtained. Using Equation 3, a new Mach number $M_{u, \text { new }}$ is calculated from the new inlet conditions. Based on the relation of the new Mach number to the Mach numbers of the previously calculated dimensionless curves, the full dimensionless curve, consisting of the new head coefficient $\psi_{\text {new }}$ and the new inlet flow coefficient $\varphi_{1, \text { new }}$, is created by a suitable curve approximation method. Lastly, the actual new operating curve, consisting of $H_{p, \text { new }}$ plotted against $\dot{V}_{\text {new }}$, is calculated from the new dimensionless curve using Equations 7 and 8

$$
\begin{gathered}
H_{p, \text { new }}=\psi_{\text {new }} \frac{U_{\text {new }}^{2}}{2} \\
\dot{V}_{\text {new }}=\varphi_{1, \text { new }} U_{\text {new }} d^{2} \frac{\pi}{4}
\end{gathered}
$$

When operating within the Mach numbers given in the design data, using this method the new polytropic head curves can be approximated within an accuracy of $3 \%$. The method does, however, have some weaknesses. It is strictly valid only if each stage of the compressor is treated separately. In addition, the accuracy of the prediction quickly deteriorates if the new dimensionless curves are extrapolated from those derived from the manufacturer data. (Ravi et al, 2015)

If the head coefficient $\psi$ is used in conjunction with the exit flow coefficient $\varphi_{3}$ instead of the inlet flow coefficient $\varphi_{1}$, the performance of each compressor stage can be presented in a single dimensionless curve for all conditions up to a critical Mach number. For Mach numbers above the critical values, the curves will be reduced and lie to the left of the curve for the lower Mach numbers. During tests performed for two compressors, both the head coefficients and efficiencies could be presented in a single curve while operating below the critical Mach numbers, even as molecular weights of the compressed gas ranged from $29 \mathrm{~g} / \mathrm{mol}$ to $365 \mathrm{~g} / \mathrm{mol}$. A central finding is that a single gas parameter does not affect the flow similarity. Rather, the combination of the parameters making up the tip speed Mach number is what counts as a flow similarity characteristic. (Sheets, 1952; Lüdtke, 2004)

To calculate $\varphi_{3}$ Equation 9 is used, where $b_{2}(\mathrm{~m})$ is the impeller blade tip height. Indices 2 and 3 refer to the impeller exit and compressor exit, respectively. The impeller diameter and blade tip height are usually provided in the manufacturer data sheets. The ratio of the static volumetric flow rate $\dot{V}_{3}$ to the inlet volumetric flow rate is given by Equation 10, from which $\dot{V}_{3}$ is easily solved. The ratio of the temperatures can be solved from Equation 11, where $m(-)$ is the polytropic temperature exponent. (Liese, 2017; Lüdtke, 2004; Sinnot 2005)

$$
\begin{gathered}
\varphi_{3}=\frac{\dot{V}_{3}}{\pi d b_{2} U} \\
\frac{\dot{V}_{3}}{\dot{V}_{1}}=\frac{T_{3}}{T_{1}} \frac{p_{1}}{p_{3}} \frac{Z_{3}}{Z_{1}}
\end{gathered}
$$

$$
T_{3}=T_{1}\left(\frac{p_{3}}{p_{1}}\right)^{m}
$$

Once the dimensionless curve consisting of $\psi$ and $\varphi_{3}$ has been constructed, the performance curve for the new operating conditions can be calculated. Equation 7 is used to calculate the new values for the polytropic head. The values for the inlet volume flow at the new process conditions can be calculated with Equation 12, where $\dot{V}_{3}$ is given by Equation 13. (Nyholm, 2020)

$$
\begin{gathered}
\dot{V}_{1}=\dot{V}_{3} \frac{T_{1}}{T_{3}} \frac{p_{3}}{p_{1}} \frac{Z_{1}}{Z_{3}} \\
\dot{V}_{3}=\varphi_{3} \pi d b_{2} U
\end{gathered}
$$

The new temperature ratio is given by Equation 14 and the new outlet pressure $p_{3}(\mathrm{~Pa})$ is given by Equation 15. The polytropic index $n(-)$ can be calculated from $\kappa$ and the polytropic efficiency $\eta_{p}(-)$ according to Equation 16. The value of the polytropic efficiency should correspond to the value of $\varphi_{3}$. Equation 17 is used in the calculation of the compressibilities, where $V_{m}$ $\left(\mathrm{m}^{3} / \mathrm{mol}\right)$ is the molar volume. (Nyholm, 2020)

$$
\begin{gathered}
\frac{T_{1}}{T_{3}}=\frac{1}{\left(\frac{p_{3}}{p_{1}}\right)^{m}} \\
p_{3}=p_{1} \times\left(\frac{n-1}{n}\right) \sqrt{\frac{H_{p}}{\frac{R}{M W} Z_{\text {avg }} T_{1}\left(\frac{n}{n-1}\right)}+1} \\
n=\frac{1}{1-\frac{\kappa-1}{\kappa \eta_{p}}} \\
Z=\frac{p V_{m}}{R T}
\end{gathered}
$$

Once $H_{p}$ and $\dot{V}_{1}$ at the new process conditions are calculated for the range of the dimensionless curve, the new operating curve is ready for use. Unfortunately, even this method is strictly applicable only for a single compression stage. A general way to describe the operation of the whole compressor in a single curve would be a great benefit for accurate dynamic simulation, but alas no such thing as an invariant performance curve seems to exist for multistage compressors. (Lüdtke, 2004)

The outlet temperature of the compressor can be calculated with Equation 11. When not operating at critical conditions, $m$ reduces to Equation 18. (Sinnot, 2005)

$$
m=\frac{\kappa-1}{\kappa \eta_{p}}
$$

For a simple approximation of the compressor power consumption $P(\mathrm{~W})$, Equation 19 can be used, where $h$ $(\mathrm{J} / \mathrm{kg})$ is the specific enthalpy of the gas, $\dot{m}(\mathrm{~kg} / \mathrm{s})$ is the mass flow and $\eta(-)$ is the general efficiency of the compressor. (Westerlund, 2009) 


$$
P=\frac{\dot{m}\left(h_{3}-h_{1}\right)}{\eta}
$$

\section{Developed dynamic model}

For a dynamic simulator used in operator training, correct and accurate behavior is vital. For a single piece of process equipment in the model, it is of central importance that both the direction and size of the dynamic response to changes in the process conditions match reality. Bearing in mind that individual process equipment are an integral part of a larger process model also accentuates the importance of the accuracy of the absolute values for e.g. flow and temperature calculated by the model, as these affect the downstream process model. As long as the dynamic behavior of the model is correct, it may be possible to use empiric correction factors to adjust the absolute process values to correct levels. However, excessive use of such corrections are likely to cause further model issues sooner or later. False behavior of both individual equipment and the total process model are severely detrimental for use of the model in both educational and engineering applications.

The simulator should, at the bare minimum, be able to run in real-time, which limits the acceptable computational complexity for any single piece of machinery or equipment in the process model. As the process model needs to be able to handle a plethora of operating conditions and upset scenarios, the compressor model should also be flexible and robust. Changes in the inlet temperature, gas composition, pressure and flowrate, rotation speed of the compressor, as well as changes in the outlet pressure, should affect the compressor operation in an appropriate manner.

As computationally heavy methods such as CFD modeling of the entire machine is not an option for dynamic simulation, the best way to accurately calculate the operation of the machine would seem to be to use the performance curves and determine how they change with varying operating conditions.

The compressor operation is characterized by the operating point, outlet temperature and consumed power, of which the operating point is most important. As such, the focus during development of the new model was mainly on calculating the operating point and describing its behavior during off-design operation.

Bearing the mentioned criteria in mind, the exit flow coefficient method appeared as the most suitable for operating curve prediction. Power and outlet temperature calculations were also implemented, but in a relatively simple manner.

The developed method starts off by determining the dimensionless operating curve for the compressor. Equations 5 and 9 were used to transform the manufacturer curves to dimensionless form. A simplified form of Equation 10 was used, without the compressibilities. Equations 11 and 15 were used for calculating the outlet pressure and temperature. In Equation 15 the inlet compressibility was used, instead of the average. The modeled compressor was modeled as a single-stage operation, so only one dimensionless curve was used for the entire machine. Calculation of $m$ was done with Equation 20. To compensate for the reduced assumption made in Equation 18, the polytropic efficiency was added. The tuning parameter $m_{\text {corr }}(-)$ was also added. This parameter is common for all the curves and can be used by the modeler to align them. (Nyholm, 2020)

$$
m=\frac{n-1}{n \eta_{p}} m_{\text {corr }}
$$

Once polynomials have been fitted to the dimensionless performance curve and the dimensionless polytropic efficiency curve, the coefficients of the polynomials are given as input to the constructed compressor model in ProsDS. The model reads the temperature, pressure, molar weight, specific heat capacity, molar flow, molar volume and enthalpy from the inlet flow. The pressure and enthalpy are read from the outlet stream. The dimensionless curve in ProsDS is defined as a set of points. The polytropic head and inlet volume flow are calculated with Equations 7 and 12. The model determines the operating point through interpolation with the help of the governing outlet pressure and writes the volume flow to the inlet stream. Using Equation 21 the compressor model then calculates and writes the temperature to the outlet stream. The compressibility was tested as a correction factor for the outlet temperature. Based on some tests in Excel, adding $Z$ to the exponent seemed to improve the accuracy of the temperature calculation at design point operation. The effect of the correction depends on the used equation of state. The power consumption was calculated with Equation 22, where $\dot{\xi}(\mathrm{mol} / \mathrm{s})$ denotes the molar flow and $h(\mathrm{~J} / \mathrm{mol})$ is the molar enthalpy of the stream. (Nyholm, 2020)

$$
\begin{gathered}
T_{3}=T_{1}\left(\frac{p_{3}}{p_{1}}\right)^{(n-1) / n Z} \\
P=\dot{\xi}\left(h_{3}-h_{1}\right)
\end{gathered}
$$

The special operating conditions surge and choke were also considered in the model, although in a more indicative manner. The limits for surge and choke were taken as the end points from the manufacturer curves If the model predicted surge the flow would drop to zero, whereas if choke was predicted the flow would be limited at a maximum value. (Nyholm, 2020)

\section{Results}

To evaluate the stability, accuracy and dynamic behavior of the constructed model, a series of test were performed in the simulator. The results were compared to both manufacturer data and process data. Differences in the calculated values of $\kappa$ and $Z$ and the values given 
by the manufacturer were noted. To determine the effect of these differences the model was tested at a number of operating points along a single operating curve. It was determined that the difference had a lowering effect on the predicted operating curve. Effects on the power consumption were also investigated. For this, both the ideal and Soave-Redlich-Kwong (SRK) equations of state were tested. The tests showed that the difference in the values increased the power consumption. If the values were given as constants matching the process data, the SRK equation of state gave better results. If the values were calculated by the simulator the ideal equation of state gave results closer to the manufacturer data. (Nyholm, 2020)

The model was also subjected to more dynamic tests to verify its stability and correct dynamic behavior. During these tests, the temperature, pressure and molecular weight of the inlet stream as well as the pressure of the outlet stream were adjusted by a ramp function in the simulator. Some issues could be observed when the values were ramped down to extremely low, abnormal levels, such as temperatures close to absolute zero or pressures close to complete vacuum. Around realistic operating regimes the model displayed realistic dynamic behavior and smooth transitions between surge, normal operation and choke. (Nyholm, 2020)

Once the validity of the concept had been proved in Excel and the stability of the constructed model ascertained with the dynamic tests, the testing could progress to assess the actual accuracy of the model by performing the process data tests. Process data of three different operating cases was available for the modeled compressor. The data of the stream inlet temperature, composition and pressure as well as outlet pressure, was fed to the test model, which then predicted the inlet volume flow, outlet temperature and compressor power consumption. The old compressor model implemented in ProsDS was tested in parallel to the newly developed model for comparison.

The first case to be tested was the shutdown operation. This case was tested twice; once with the ideal equation of state and once with the SRK equation of state. The predicted mass flow and temperature showed a smaller offset form the process data when using the ideal equation of state, although the power consumption had a somewhat larger offset. As the ideal equation overall gave better results it was used in the other tests as well.

During the first test case it was clearly visible that the mass flow predicted by the new model was an improvement. At conditions close to normal operation the predicted flow lies slightly above the measured flow. As the process conditions diverge from normal operation the offset between the curves can be seen to increase. The reason behind this would seem to be increasing inaccuracies in the predicted operating curves, as the predictions for some of the curves will lie more to the left of the manufacturer performance curves when moving from the rated operating conditions towards conditions resembling the initial circulation case. The shape of the trend clearly followed the process data better than the predictions of the old model. The oscillations seen towards the end of the predicted curve are due to the model balancing on the verge of surge.

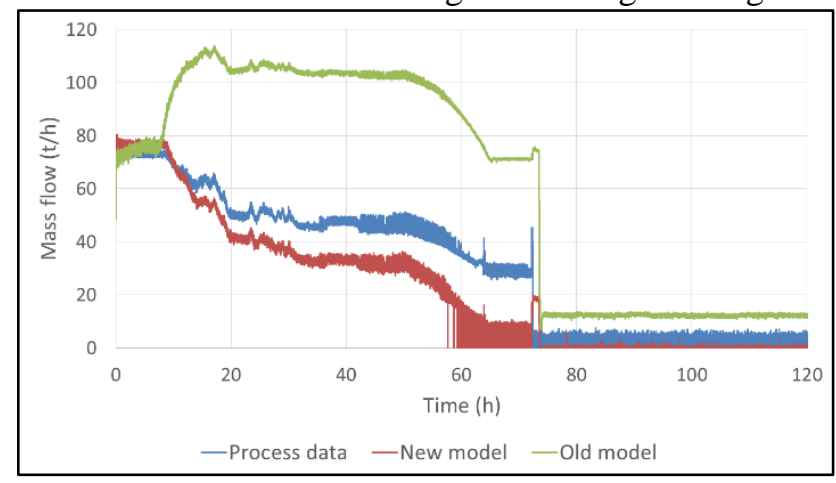

Figure 1. Test result for the suction mass flow of the shutdown case using the ideal equation of state.

As can be seen in Figure 2 the temperature calculation of the old model works quite well. The shape of the predicted curve is very similar to that of the process data. During normal operation, this prediction is slightly better than the new model's prediction, but as the conditions diverge from normal operation the new model gives a better prediction. The predicted curves have been cut as the machine is turned off, since they are no longer accurate after that point. (Nyholm, 2020)

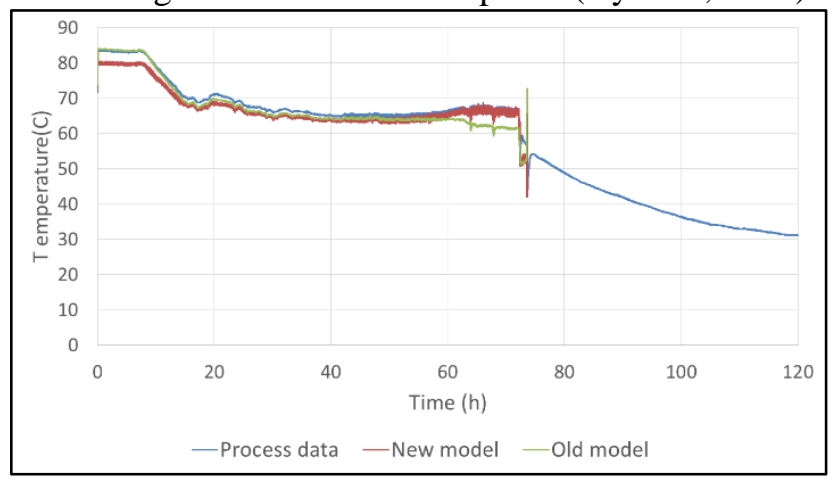

Figure 2. Test result for the outlet temperature of the shutdown case.

The predicted electric current initially lies above the process data with a bigger offset than in the SRK test case but approaches the process data curve towards the end of the operation, as seen in Figure 3. The predicted current of the old model differs completely from the process data. (Nyholm, 2020) 


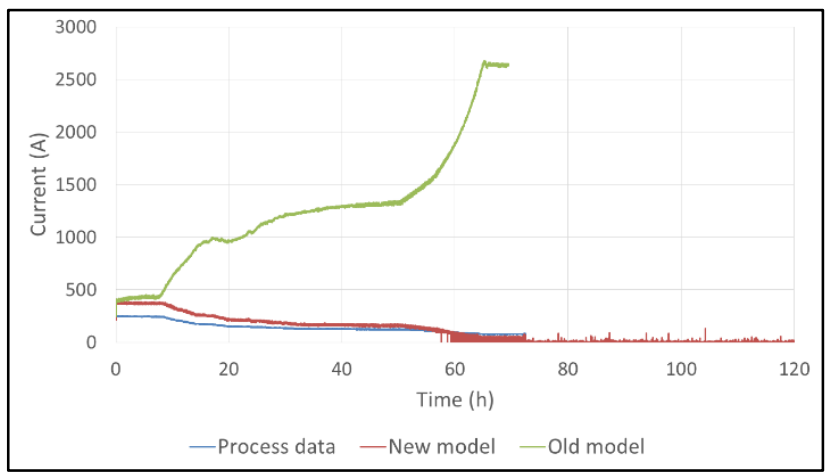

Figure 3. Test result for electric current of the shutdown case.

The test results of the predicted mass flow for the normal operation case and the startup case are shown in Figure 4 and Figure 5. The new model gives a clearly improved prediction compared to the old model, although some discrepancy is visible in the startup case, where the model is predicting surge for some time at the start. The predictions of the outlet temperature and electric current behaved in a similar manner as in the shutdown case. (Nyholm, 2020)

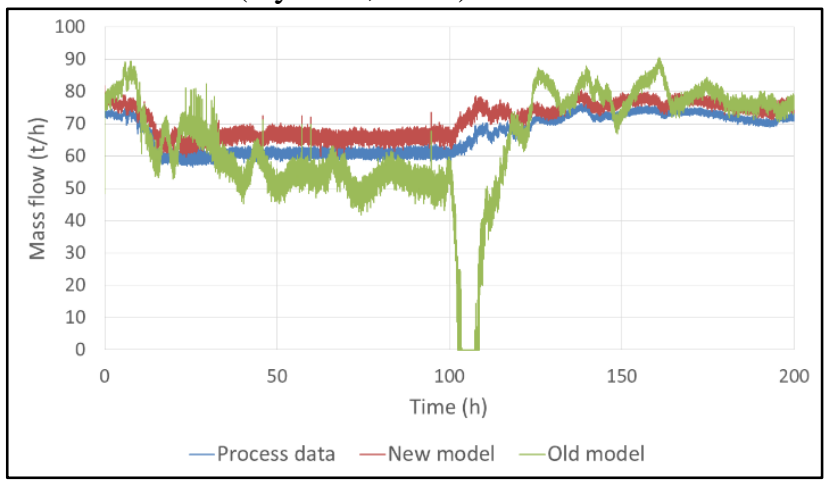

Figure 4. Test result for the suction mass flow of the normal operation case.

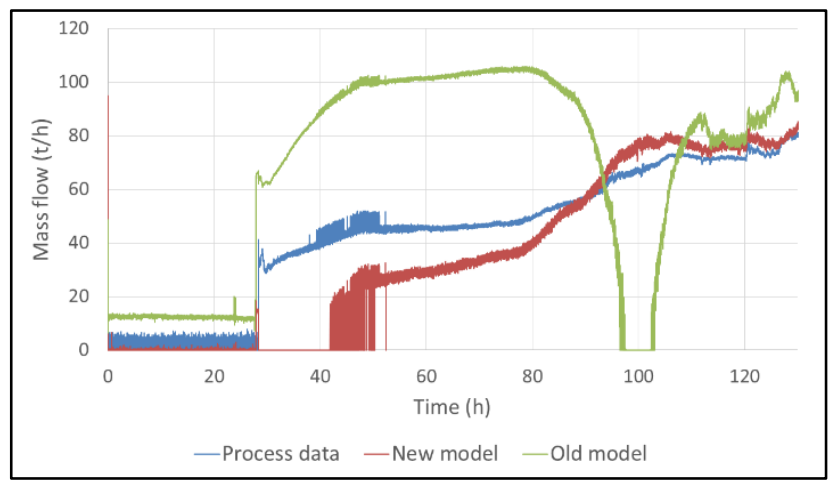

Figure 5. Test result for the suction mass flow of the startup case.

\section{Discussion}

\subsection{Benefits}

The benefits of an OTS during the design and commissioning phase of a project include functions for testing the design of control and safety automation systems and functionality of operator user interface layouts, virtual testing of process design and changes and planning of safe startups and shutdowns. When used for operator training, the operators can safely be trained for coping with any fault situation. Additionally they can be trained in how to operate the facility for maximal production efficiency. Increased availability and reduced downtime of the process plant are direct benefits. (Qvist et al, 2018)

The improved compressor model will benefit the NAPCON Simulator OTS by increasing the accuracy of the process models especially during shutdown and startup operations. A more accurate performance of the models during these operations is crucial for realistic response during training scenarios of such situations.

The new method also allows for a faster modeling of the machines, as the need for external calculations are reduced. The person doing the modeling will not have to search for and tune the correlations between the process parameters and compressor performance as extensively as previously, as they are already included in the model.

\subsection{Future development}

Based on the results of the various tests performed for the developed model several areas of improvement were identified. These subjects mainly relate to the operating curve, outlet temperature calculation and power calculation. Based on some preliminary tests, the most promising way to address the greater inaccuracies of the model, namely the offset of the flow prediction, appears to be implementing a method for scaling the polytropic efficiency to match the operating conditions. (Nyholm, 2020)

\section{Conclusions}

A new dynamic simulation model for centrifugal compressors was constructed using dimensionless operating curves. With the help of a tuning parameter the method could be used for a multi-stage compressor with promising results.

The developed model is stable over a wide range of operating conditions, supporting the feasibility of the selected method of modeling the compressor operation. When examining the tests and figures of section 4 , it can be found safe to claim that modeling of the compressor flow and required electric current were substantially improved by the developed model.

The flow and outlet temperature calculations of the new model performed better when using the ideal 
equation of state, while the electric current calculation fared better when the SRK equation of state was used. When using the SRK method the outlet temperature predicted by the developed model seemed better compared to the old model's prediction. When the ideal equation of state was used, however, the outlet temperature predicted by the old model described the process data more accurately for the most part of the test. The accuracy of the new model's temperature prediction was better only shortly before the machine was shut off or shortly after it was turned on.

When studying the graphs in section 4, the possibility of inaccuracies and various uncertainties pertaining to both the manufacturer data and the measured process data should be kept in mind. Albeit the developed model overall appears to be a clear improvement, it still suffers from certain shortcomings and requires further development to overcome these issues.

\section{References}

Eric Liese, Stephen E. Zitney. The Impeller Exit Flow Coefficient as a Performance Map Variable for Predicting Centrifugal Compressor Off-Design Operation Applied to a Supercritical CO2 Working Fluid. ASME Turbo Expo 2017: Turbomachinery Technical Conference and Exposition, Vol 9, 2017.

Klaus H. Lüdtke. Process Centrifugal Compressors. SpringerVerlag Berlin Heidelberg GmbH. 2004.

J. Fredrik Nyholm. Improved modeling of rotary momentum mass transport equipment, M.Sc. thesis, Åbo Akademi University, 2020.

Frank Pettersson, Hans Skrifvars, Kurt Lundqvist, Kaj Fagervik, Tapio Westerlund. Ett övningsarbete $i$ pumpdimensionering. Åbo Akademi University. 1990.

Pekka Qvist, Ari Vepsäläinen and Prateek Agarwal. Develop competence in the process industry with a digitalized operator learning path. Hydrocarbon Processing, 97(7), 2018

Arjun Ravi, Lukasz Sznajder, Ian Bennett. Compressor map prediction tool. IOP Conference Series: Materials Science and Engineering, Vol 90, 2015. doi:10.1088/1757899X/90/1/012042

H. E. Sheets. Nondimensional Compressor Performance for a Range of Mach Numbers and Molecular Weights. Transactions of the ASME, 74(1):93-102, 1952.

R. K. Sinnot. Coulson \& Richardson's Chemical Engineering Volume 6: Chemical Engineering Design. Elsevier Butterworth-Heinemann. 2005.

Tapio Westerlund. Anläggnings- och systemteknik. Tekniska fakulteten vid Åbo Akademi. 2009. 